\title{
Correlated Traffic Modelling: Batch Renewal and Markov Modulated Processes*
}

\author{
Rod Fretwell and Demetres Kouvatsos \\ Computer Systems Modelling Research Group, \\ University of Bradford \\ Bradford BD7 1DP, U.K. \\ email \{R.J.Fretwell,D.D.Kouvatsos\}@comp.brad.ac.uk
}

\begin{abstract}
The usual measures of traffic correlation do not determine the traffic process completely. Consequently there may be a number of traffic processes which induce the same correlation but which differ in other features and so differ in impact on performance.

An experiment was devised to distinguish the effects of traffic process features from the effects due to the correlation alone. The batch renewal process is the ideal of a correlated traffic process and so provides the standard (in terms of buffer congestion, etc.) against which the performance impact of any other correlated traffic process may be compared.

This paper presents results of the experiment as performed upon the Markov modulated processes, together with further analysis to assess the applicability of the findings, and concludes that a Markov modulated process is likely to yield more optimistic estimates of cell loss rate, delay, etc., than are attributable to the traffic correlation alone, especially in simple models with a low number of phases.
\end{abstract}

\section{INTRODUCTION - BACKGROUND AND MOTIVATION}

A perennial problem in performance modelling and evaluation is the choice of most appropriate model process. Whether the task be to fit a model to measurements from a real process or to investigate (algebraically, numerically or by simulation) the effect of varying some characteristic, the choice of model may be crucial. In modelling a real process, a poorly chosen model has little predictive value. In analysis, a poor choice of model is one which has features other than the characteristic which is to be studied: it is possible that the effect of other features could dominate the results of analysis. In very few

${ }^{*}$ This work is supported in part by the Engineering and Physical Sciences Research Council (EPSRC), UK, under grant GR/K/67809

OIFIP 1997. Published by Chapman \& Hall 
circumstances is the best choice determinate and generally agreed: a) if the inter-event time be memoryless then its distribution is negative exponential (a Poisson process) in continuous time or geometric in discrete time; b) if the process exhibits no correlation (neither of counts nor of intervals) then its inter-event time distribution is Generalised Exponential or Generalised Geometric, provided that the first two moments exist. More generally, however, choice of model process depends upon expertise and experience and, ultimately, upon preference.

Traffic correlation is widely recognised as being an important issue, especially in ATM performance, and the literature contains a variety of processes which may be used to model correlated traffic. The analytic models which have been most frequently employed are the Markov modulated processes (MMPP, IPP, MMBP, IBP, MMBBP, etc.), MAP's, BMAP's, etc., and the semi-Markov or Markov renewal processes. The impact of correlation has been well known, in general terms (Sriram and Whitt 1986), for some time and appropriate metrics for correlation (indices of dispersion, etc. (Gusella 1991)) are established and in general use. However, to investigate the effects of correlation or to fit a model to traffic measurements was to face the problem of choosing, from those available in the literature, the class of model (e.g. a Markov modulated process) and its features (e.g. the number of phases in the modulating chain and the type of process to be modulated). Fitting traffic measurements also necessitated selecting a method of estimating the parameters. Sriram and Whitt (1986), Heffes and Lucantoni (1986) and Gusella (1991) each propose a different procedure for fitting a 2-phase MMPP.

Discovery of the batch renewal process (Kouvatsos and Fretwell 1994) provided means to investigate the effect of traffic correlation independent of any other characteristic. Because the batch renewal process is completely determined by count and intervals correlation at all lags, it can (in principle*) be constructed to match traffic measurements exactly (Kouvatsos and Fretwell 1996). Further, for correlated traffic (viewed as a 2-dimensional wide sense stationary process) the batch renewal process is the least biased choice given the usual measures of correlation and so enables the effects of traffic correlation alone to be quantified precisely (Kouvatsos and Fretwell 1995).

Because the measures of correlation are statistics, which do not define the traffic completely, it is possible that two or more models (with appropriate parameterisation) might generate traffic with the same measures of correlation. It is of interest to distinguish the impact of features peculiar to each type of model. The approach adopted in this paper is to compare the impact of traffic from a particular model with the effects due to the correlation alone, i.e. by reference to the batch renewal process constructed to have identical correlation, both of counts and of intervals, at all lags.

Section 2 presents the notation, conventions and formulae which are used in

${ }^{*}$ Some combinations of count and intervals correlation yield a batch renewal process which has improper distributions. 
the paper and distinguishes what is meant here by "Markov modulated process" from other models of correlated traffic. Section 3 describes the design of the main experiment and gives illustrative results. Generalisation of the findings is discussed in Section 4: that they be plausible is considered by reference to a stereotype model; that the experimental results were not affected by unwitting bias is supported by a Monte Carlo experiment. Conclusions and directions for future work are given in Section 5 .

\section{PRELIMINARIES}

\subsection{Notation}

In discrete time systems, events occur at discrete points in time, called epochs. The intervals between epochs are called slots. The convention adopted in this paper is that epochs and slots are both numbered in sequence so that slot $t$ is the interval between epoch $t-1$ and epoch $t$ and so that epoch $t$ is the epoch which ends slot $t$ and begins slot $t+1$.

The following notation is used consistently throughout this paper.

$\mathbb{Z}, \mathbb{N}_{0}, \mathbb{N}_{+}$the sets of integers, of non-negative integers and of positive integers

$\{c(t): t \in \mathbb{Z}\}$ the random sequence of the count $c(t) \in \mathbb{N}_{0}$ of the number of arrivals at epoch $t$

$\{x(n): n \in \mathbb{Z}\}$ the random sequence of the interval $x(n) \in \mathbb{N}_{0}$ between the $(n-1)$ th individual arrival and the $n$th individual arrival

MMBBP Markov modulated batch Bernoulli process

$\phi(t)$ the phase of the modulating chain of the MMBBP in slot $t$

$\boldsymbol{\Phi}=\left(\phi_{i j}\right): i, j=1, \ldots, J$ the MMBBP phase transition matrix of degree $J$ with $\phi_{i j}=\mathrm{P}[\phi(t+1)=j \mid \phi(t)=i]$ independent of $t$

$\phi=\left(\phi_{1}, \ldots, \phi_{J}\right)$ the stationary distribution vectorof $\boldsymbol{\Phi}$

1 the $J$-element column vector of 1 's

$\left\{\lambda_{i}(n): i=1, \ldots, J, n \in \mathbb{N}_{0}\right\}$ probability mass function ( $\left.p m f\right)$ for the probability $\lambda_{i}(n)$ that the MMBBP generate the count of $n=0,1, \ldots$ individual arrivals when in phase $i$

$\Lambda_{i}(z)=\sum_{n=0}^{\infty} \lambda_{i}(n) z^{n}$ probability generating function for counts in phase $i$

$\boldsymbol{\Lambda}(z)=\operatorname{diag}\left(\Lambda_{1}(z), \ldots, \Lambda_{J}(z)\right)$

$\boldsymbol{\Lambda}=\boldsymbol{\Lambda}^{\prime}(1)$

$\lambda=\phi \Phi 1$ overall mean rate of arrivals 
$\left\{I_{t}: t \in \mathbb{N}_{+}\right\}$index of dispersion for counts (IDC),

$$
I_{t}=\operatorname{Var}\left[\sum_{r=1}^{t} c(r)\right] / \mathrm{E}\left[\sum_{r=1}^{t} c(r)\right]
$$

$\left\{J_{n}: n \in \mathbb{N}_{+}\right\}$index of dispersion for intervals (IDI),

$$
J_{n}=n \operatorname{Var}\left[\sum_{r=1}^{n} x(r)\right] / \mathrm{E}\left[\sum_{r=1}^{n} x(r)\right]^{2}
$$

$K(\omega)$ generating function for count covariances and IDC

$L(z)$ generating function for intervals covariances and IDI

$P(z)$ generating function for MMBBP/D/1 queue length distribution (q.l.d.)

$\left\{a(t): t \in \mathbb{N}_{+}\right\} \quad p m f$ of intervals between batches in the batch renewal process

$\left\{b(n): n \in \mathbb{N}_{+}\right\} \quad p m f$ of batch sizes in the batch renewal process

$A(\omega)=\sum_{t=1}^{\infty} a(t) \omega^{t}$ probability generating function for intervals between batches in the batch renewal process

$B(z)=\sum_{n=1}^{\infty} b(n) z^{n}$ probability generating function for batch sizes in the
batch renewal process

$\delta_{i j} \quad$ Kronecker delta

\subsection{Simultaneity Considerations}

At each epoch there may be a number of simultaneous events.

In the MMBBP/D/1 and MMBBP/D/1/N queues these events are: change of phase in the modulating chain which underlies the MMBBP; generation of arrivals, according to the phase of the MMBBP; departure from the queue, when the server was busy in slot which is terminated by the epoch. The logical sequence of those events must be determined.

- Given that the modulating chain be in phase $i$ during slot $t$, the probability of there being $n$ arrivals at epoch $t$ and the modulating chain being in phase $j$ in slot $t+1$ is

$$
\begin{aligned}
\mathrm{P}[c(t)=n, \phi(t+1) & =j \mid \phi(t)=i] \\
& =\mathrm{P}[c(t)=n \mid \phi(t+1)=j, \phi(t)=i] \mathrm{P}[\phi(t+1)=j \mid \phi(t)=i] .
\end{aligned}
$$

The convention adopted in this paper is that generation of arrivals logically precede phase switch. Consequently,

$$
\begin{aligned}
\mathrm{P}[c(t)= & n \mid \phi(t+1)=j, \phi(t)=i] \mathrm{P}[\phi(t+1)=j \mid \phi(t)=i] \\
& =\mathrm{P}[c(t)=n \mid \phi(t)=i] \mathrm{P}[\phi(t+1)=j \mid \phi(t)=i] \\
& =\lambda_{i}(n) \phi_{i j} .
\end{aligned}
$$

The alternate convention, that phase switch logically precede generation of 
arrivals, would have given

$$
\mathrm{P}[c(t)=n, \phi(t+1)=j \mid \phi(t)=i]=\lambda_{j}(n) \phi_{i j} .
$$

Results, such as measures of traffic correlation and queue length distribution, are not affected by the choice of convention. However, the choice does affect the details of their derivations.

- If, at an epoch, new arrivals may occupy places which are being vacated by departures at that epoch then, logically, the departures precede arrivals. This convention, known as departures first (DF), is that adopted in this paper. The alternate convention, in which new arrivals may not occupy places which are to be evacuated by departures at that epoch, is known as arrivals first (AF).

The choice of convention affects statistics such as blocking probability and distributions of queue length and waiting time in finite buffer queues. However, the difference in effect of AF and DF declines with increasing buffer size. The choice of AF or DF does not affect results for the infinite buffer queue.

\subsection{MMBBP/D/1 Queues}

The stationary probability $p(n, j)$ that, in any slot, there be $n$ customers in the queue or in service and the modulating chain of the MMBBP be in phase $j$ obviously satisfies

$$
p(n, j)=\sum_{i=1}^{J}\left(p(0, i) \lambda_{i}(n)+\sum_{k=0}^{n} p(k+1, i) \lambda_{i}(n-k)\right) \phi_{i j}
$$

for $n=0, \ldots, N-1$, and

$$
p(N, j)=\sum_{i=1}^{J}\left(p(0, i) \sum_{r=N}^{\infty} \lambda_{i}(r)+\sum_{k=0}^{N-1} p(k+1, i) \sum_{r=N-k}^{\infty} \lambda_{i}(r)\right) \phi_{i j}
$$

Equation (1) implies that, for each state $(n, j), n=0, \ldots, N, j=1, \ldots, J$, $p(n, j)$ is a linear function of the $p(0,1), \ldots, p(0, J)$. In particular, if (as is usually the case) the phase transition matrix $\Phi$ be nonsingular with inverse $\Phi^{-1}=\left(\phi_{i j}^{-1}\right)$, then equation (1) yields

$$
p(1, i)=\sum_{j=1}^{J} p(0, j) \phi_{i j}^{-1}-p(0, i) \lambda_{i}(0)
$$


(using $n=0$ in eq.(1)) and (by substituting $n+1 \rightarrow n$ ) the recurrence relationship for $n=2, \ldots, N$

$$
p(n, i)=\sum_{j=1}^{J} p(n-1, j) \phi_{i j}^{-1}-p(0, i) \lambda_{i}(n-1)-\sum_{k=1}^{n-1} p(k, i) \lambda_{i}(n-k) .
$$

The expression of $p(n, j)$ as a linear function of the $p(0,1), \ldots, p(0, J)$ is not dependent upon the capacity $N$ (although the actual values of the $p(0,1), \ldots, p(0, J)$ do depend upon $N)$. Consequently, the queue length distribution of the finite buffer MMBBP/D/1/N queue is the same linear function of the $p(0,1), \ldots, p(0, J)$ as the queue length distribution of the infinite buffer MMBBP/D/1 queue. In particular, if the infinite buffer MMBBP/D/1 queue length distribution be a weighted sum of geometrics then the finite buffer $\mathrm{MMBBP} / \mathrm{D} / 1 / \mathrm{N}$ queue length distribution is a weighted sum of the same geometrics. Only the weights vary with the buffer size $N$.

For the infinite buffer queue, equation (1) leads to the vector generating function

$$
\begin{aligned}
\boldsymbol{P}(z) & =\sum_{n=0}^{\infty}(p(n, 1), \ldots, p(n, J)) z^{n} \\
& =\left(\frac{z-1}{z} \boldsymbol{P}(0)+\frac{1}{z} \boldsymbol{P}(z)\right) \boldsymbol{\Lambda}(z) \boldsymbol{\Phi}
\end{aligned}
$$

and, therefore,

$$
P(z)=\boldsymbol{P}(z) \mathbf{1}=(z-1) \boldsymbol{P}(0)(z \mathbf{I}-\boldsymbol{\Lambda}(z) \Phi)^{-1} \boldsymbol{\Lambda}(z) \mathbf{1}
$$

\subsection{MMBBP Correlation}

The usual measures of traffic autocorrelation are either the sequence of count covariances and the sequence of intervals covariances or, equivalently, the index of dispersion for counts (IDC) and the index of dispersion for intervals (IDI). The following generating function expressions incorporate the well known relationships between those measures.

$$
\begin{aligned}
K(\omega) & \triangleq \frac{1}{\lambda}\left(\operatorname{Var}[c(t)]+2 \sum_{\ell=1}^{\infty} \operatorname{Cov}[c(t), c(t+\ell)] \omega^{\ell}\right) \\
& \equiv(1-\omega)^{2} \sum_{t=1}^{\infty} t I_{t} \omega^{t-1} \\
L(z) & \triangleq \lambda^{2}\left(\operatorname{Var}[x(n)]+2 \sum_{\ell=1}^{\infty} \operatorname{Cov}[x(n), x(n+\ell)] z^{\ell}\right) \\
& \equiv(1-z)^{2} \sum_{n=1}^{\infty} n J_{n} z^{n-1}
\end{aligned}
$$


A sufficient condition for existence of the sums on the unit disk $(|\omega| \leq 1$ or $|z| \leq 1$, as appropriate) is that the covariances be Cesaro summable. Then $K(1)=I_{\infty}=J_{\infty}=L(1)$. That condition is satisfied for the processes which are considered in this paper.

For the MMBBP, expressions (4) and (5) have the equations.

$$
\begin{aligned}
& K(\omega)=\frac{1}{\lambda} \phi \Lambda^{\prime \prime}(1) \mathbf{1}+1+\frac{2}{\lambda} \omega \phi \Lambda^{\prime}(1) \Phi(\mathbf{I}-\omega \Phi)^{-1} \Lambda^{\prime}(1) \mathbf{1}-\lambda \frac{1+\omega}{1-\omega} \\
& L(z)=2 \lambda \phi(\mathbf{I}-\Lambda(z) \Phi)^{-1} \Lambda(z) \mathbf{1}+\lambda-\frac{1+z}{1-z}
\end{aligned}
$$

Derivation of equation (6) follows from consideration of

$$
\begin{aligned}
\mathrm{E}[c(t) c(t+\ell)] & =\sum_{i, j=1}^{J} \mathrm{E}[c(t+\ell) \mid \phi(t+\ell)=j] \mathrm{P}[\phi(t+\ell)=j \mid \phi(t)=i] \\
& =\sum_{i, j=1}^{J} \lambda_{j} \phi_{i j}^{\ell} \lambda_{i} \phi_{i} \\
& =\phi \Lambda^{\prime}(1) \Phi^{\ell} \Lambda^{\prime}(1) \mathbf{1}
\end{aligned}
$$

Equation (7) may be derived by considering an arbitrary arrival and its $n$ immediate successors (see Figure 1), as in the following outline derivation.

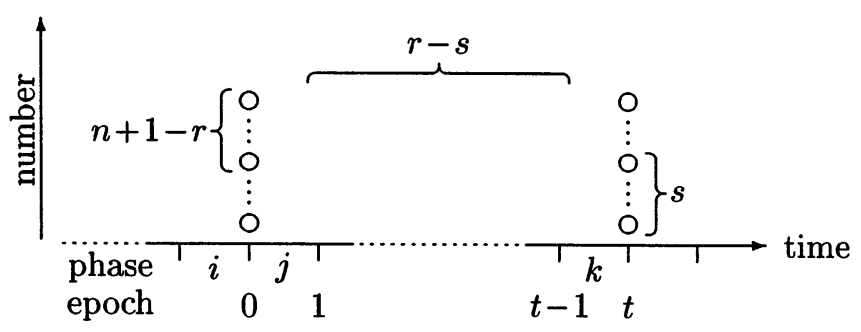

Figure 1 Ways in which $n$ intervals may span $t$ slots $(t \geq 1)$

1. the probability that an arbitrarily selected arrival be generated by phase $i$ is $\frac{\lambda_{i} \phi_{i}}{\lambda}$,

2. the probability that the arrival be in a batch of size $u$ is $\frac{u \lambda_{i}(u)}{\lambda_{i}}$ and

3. the probability that the arrival be at any particular position in the batch of size $u$ is $\frac{1}{u}$.

So the probability that $n$ intervals between $n+1$ successive arrivals span $t \geq 1$ 
slots is

$$
\sum_{i, j, k} \sum_{r=1}^{n} \sum_{s=1}^{r} \frac{\lambda_{i} \phi_{i}}{\lambda} \sum_{u=n+1-r}^{\infty} \frac{u \lambda_{i}(u)}{\lambda_{i}} \frac{1}{u} \phi_{i j} \nu(r-s, t-1, k ; j) \sum_{v=s}^{\infty} \lambda_{k}(v)
$$

where $\nu(n, t, j ; i)$ is the probability that the MMBBP, begining in phase $i$, generate $n$ arrivals in $t$ epochs and then switch to phase $j$. Clearly, $\nu(n, t, j ; i)$ is generated by

$$
\sum_{n=0}^{\infty} \sum_{t=0}^{\infty} \nu(n, t, j ; i) z^{n} \omega^{t}=(\mathbf{I}-\omega \Lambda(z) \Phi)_{i j}^{-1} .
$$

The probability that $n$ intervals be contained in the same batch (i.e. $t=0$ ) is

$$
\sum_{i} \frac{\lambda_{i} \phi_{i}}{\lambda} \sum_{u=n+1}^{\infty} \frac{u \lambda_{i}(u)}{\lambda_{i}} \frac{u-n}{u}
$$

which, combined with equation (8), yields

$$
\begin{aligned}
T(z, \omega) & =\sum_{n=1}^{\infty} \sum_{t=0}^{\infty} \mathrm{P}\left[\sum_{r=1}^{n} x(r)=t\right] z^{n} \omega^{t} \\
& =\frac{z}{1-z}-\frac{1}{\lambda} \frac{z}{(1-z)^{2}}(1-\omega) \phi(\mathrm{I}-\mathbf{\Lambda}(z) \Phi)(\mathrm{I}-\omega \mathbf{\Lambda}(z) \Phi)^{-1} \mathbf{1}
\end{aligned}
$$

Hence,

$$
\begin{aligned}
L(z) & =(1-z)^{2} \sum_{n=1}^{\infty} n J_{n} z^{n-1}=\frac{(1-z)^{2}}{z} \sum_{n=1}^{\infty} n \frac{n \operatorname{Var}\left[\sum_{r=1}^{n} x(r)\right]}{\left(\frac{n}{\lambda}\right)^{2}} z^{n} \\
& =\frac{(1-z)^{2}}{z} \lambda^{2}\left(\left.\frac{\partial^{2} T}{\partial \omega^{2}}\right|_{\omega=1}+\sum_{n=1}^{\infty}\left(\frac{n}{\lambda}-\left(\frac{n}{\lambda}\right)^{2}\right) z^{n}\right) \\
& =2 \lambda \phi(I-\Lambda(z) \Phi)^{-1} \Lambda(z) 1+\lambda-\frac{1+z}{1-z}
\end{aligned}
$$

\subsection{Least Biased Choice of Process}

Consider a 2-dimensional random sequence $\{\xi(s), \kappa(s): s \in \mathbb{Z}\}$ in which $\kappa(s)$ is the number of arrivals in batch $s$ and $\xi(s)$ is the number of slots between batch $s-1$ and batch $s$. The $\xi(\cdot)$ and the $\kappa(\cdot)$ can assume strictly positive integer values only.

For any given realisation of $\{\xi(s), \kappa(s): s \in \mathbb{Z}\}$ it is possible to determine completely two related sequences: the sequence $\{c(t): t \in \mathbb{Z}\}$ of counts and the sequence $\{x(n): n \in \mathbb{Z}\}$ of intervals between individual arrivals. Indeed, complete knowledge of any one of the sequences $\{\xi(s), \kappa(s)\},\{c(t)\},\{x(n)\}$, provides complete knowledge of both the other two sequences. 
However, given partial knowledge only of a process, the problem is to make the least biased choice from candidate models, i.e. to identify a model which is consistent with all the information which is known and with either no other assumptions or, at worst, assumptions which minimally prejudge the outcome. How, for example, from the class of Markov modulated processes, should the number of phases of the modulating Markov chain be chosen or the type of distribution which is to be modulated?

It is known (Kouvatsos and Fretwell 1995) that, when only the covariances of the counts and of the intervals are known, the least biased choice of process $\{\xi(s), \kappa(s)\}$ is such that the $\xi(s)$ are independent and identically distributed (i.i.d.) and the $\kappa(s)$ also are i.i.d. This type of process is called a batch renewal process.

Clearly, a batch renewal process is completely defined by two constituent probability distributions: the distribution of the intervals between successive batches and the distribution of the batch sizes. However, a batch renewal process is equally well defined by the set of covariances of counts at all lags and the set of covariances of intervals (between individual arrivals) at all lags or, equivalently, by the indices of dispersion. The size (cardinality) of each of these sets is infinite.

Equations (9) and (10)

$$
\begin{gathered}
K(\omega)=b\left(C_{b}^{2}+\frac{1+A(\omega)}{1-A(\omega)}-\frac{1}{a} \frac{1+\omega}{1-\omega}\right) \\
L(z)=b\left(C_{a}^{2}+\frac{1+B(z)}{1-B(z)}-\frac{1}{b} \frac{1+z}{1-z}\right)
\end{gathered}
$$

express the generating functions for count and intervals covariances in terms of the probability generating functions for the intervals between successive batches and the distribution of batch sizes in a batch renewal process (see Kouvatsos and Fretwell 1994, 1995 or 1996). For any functions $K(\omega), L(z)$ such that $K(1)=L(1)$, i.e. for any Cesaro summable sets of count covariances and intervals covariances, this pair of equations can be solved for $A(\omega)$ and $B(z)$. In this sense, there is a batch renewal process for any specified sets of count covariances and intervals covariances*.

\subsection{MMBBP and Related Process Models}

In all other sections of this paper, the term "Markov modulated process" is meant to signify one of the models which commonly is called "Markov modulated ..." (MMBP, etc.) or which is a special case of such models (SBP, IBP, etc.) as opposed to any other model (DBMAP, etc.) which could

\footnotetext{
*It should be noted, however, that the resulting forms for $A(\omega)$ and $B(z)$ may not necessarily correspond to proper probability mass functions for some combinations of count covariances and intervals covariances.
} 
feasibly be represented as a Markov modulated process. Thus a typical Markov modulated process would have a relatively small number of phases and the distribution of counts in each phase would have a relatively simple form. In the design of the experiments reported in sections 3 and 4 , heavy tailed distributions were excluded from consideration, as also were strictly cyclic modulating chains.

In general, a discrete time batch renewal process can be represented as a pathological MMBBP with infinite number of phases. In this model, the phase number is the number of slots remaining before the next batch. Arrivals are generated in phase 1 only. Then, if the $p m f$ of the interval between batches be $a(t), t=1,2, \ldots$, the components $\phi_{i j}, i, j=1,2, \ldots$, of the phase transition matrix are

$$
\phi_{i \jmath}= \begin{cases}a(j) & i=1, j=1,2, \ldots \\ 1 & j=i-1, i=2,3, \ldots \\ 0 & \text { otherwise }\end{cases}
$$

and, if the $p m f$ of the batch sizes be $b(n), n=1,2, \ldots$, the $p m f \lambda_{\imath}(n)$ of counts (number of arrivals generated at each epoch) in phase $i$ is

$$
\lambda_{i}(n)= \begin{cases}b(n) & i=1, n=1,2, \ldots \\ 1 & i=2,3, \ldots, n=0 \\ 0 & \text { otherwise }\end{cases}
$$

In a similar way, a discrete time Markov renewal process (or semi-Markov process) may be represented as a MMBBP with infinite number of phases for each phase of the Markov renewal process.

In a discrete time batch Markovian additive process (DBMAP) the distribution of counts at an epoch depends upon the phase transition. The conventional notation is

$$
\mathrm{P}[c(t)=n, \phi(t+1)=j \mid \phi(t)=i]=d_{i j}(n) .
$$

A $J$-phase DBMAP may be represented as a $J^{2}$-phase MMBBP by expanding each phase $i$ of the DBMAP into $J$ phases $i_{1}, \ldots, i_{J}$ in the MMBBP and setting

$$
\phi_{i_{k} j_{\ell}}=\delta_{k j} \sum_{n=0}^{\infty} d_{j \ell} \quad \text { and } \quad \lambda_{i_{j}}(n)=d_{i j}(n)
$$

\section{THE FIRST EXPERIMENT}

This section addresses the design of the experiment and presents typical results. The first part is applicable generally to any class of traffic process. The second focuses upon the the Markov modulated processes. Sample results are presented graphically. 


\subsection{General Considerations}

The essence of the experiment is to compare the effects of the traffic process under consideration with those due to the traffic correlation alone. In the context of ATM the interesting effects are the cell loss rate, waiting time, jitter, buffer occupancy, etc. in a queue which is served at one cell per slot. All those statistics are related to the queue length distribution.

In the experimental model the queue buffer should have infinite capacity at least for the initial investigation into a given traffic process - because if the buffer be "too small" buffer congestion and other measures of interest are

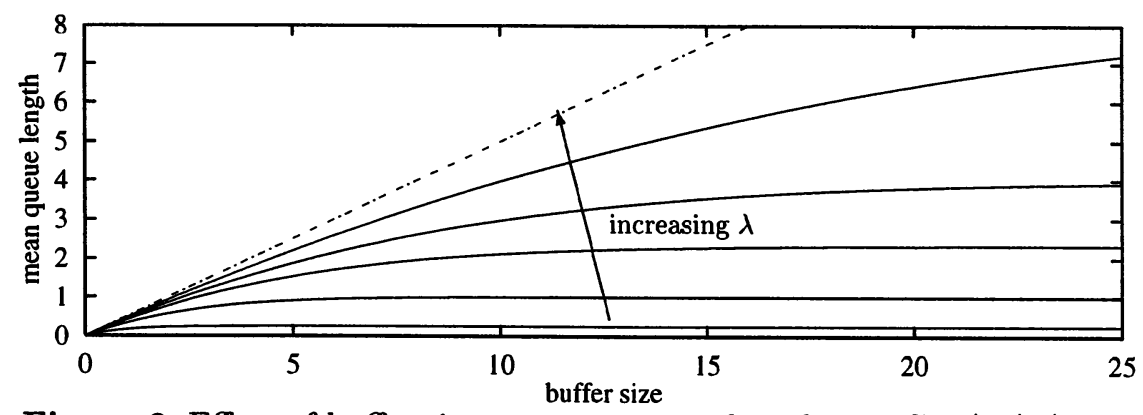

Figure 2 Effect of buffer size on mean queue length in a Geo/D/1/N queue for mean intensity $\lambda=0.2,0.5,0.7,0.8,0.9,1.0$

largely determined by the buffer size. (Figure 2 provides a simple example of the effect of buffer size and illustrates that the meaning of "small" generally depends upon traffic characteristics.) In particular, manifestation of long term dependence is limited by buffer size (see Sriram and Whitt (1986) and Kouvatsos and Fretwell (1995)). However, the magnitudes of cell loss rate, etc., in the finite buffer can be estimated from the infinite capacity queue length distribution.

So the experiment reduces to comparing the queue length distributions of two infinite capacity queues, each served deterministically at one cell per slot: the first queue is fed by the traffic process under consideration; the other queue is fed by a process which has 1) the same count correlation and interval correlation identical at all lags and 2) nothing else, i.e. a batch renewal process.

The batch renewal process is, in information theoretic terms, the least biased choice of traffic process given only the customary measures of correlation (e.g. indices of dispersion) (Kouvatsos and Fretwell 1995). In the batch renewal process there is no semblance of burst structure or, indeed, of any other feature other than correlation. The batch renewal process may be described fairly as "pure correlation".

The remaining design issues concern selection of representative members of the class of traffic processes such that the experiment should yield the most information. Ideally, the results should be applicable to the complete class. 


\subsection{Markov Modulated Processes}

The most general type of Markov modulated process in discrete time is the MMBBP. Other types of Markov modulated processes are special cases of the MMBBP or continuous time analogues.

\section{Batch Size Distributions}

It was decided to restrict consideration to geometrically bounded distributions which, for all practical purposes, means weighted sums of geometric distributions.

In order to permit the most extreme behaviour of the MMBBP, the batch Bernoulli process should comprise just two geometric components (also known as phases). Figure 3 is intended to illustrate how generally, in a distribution
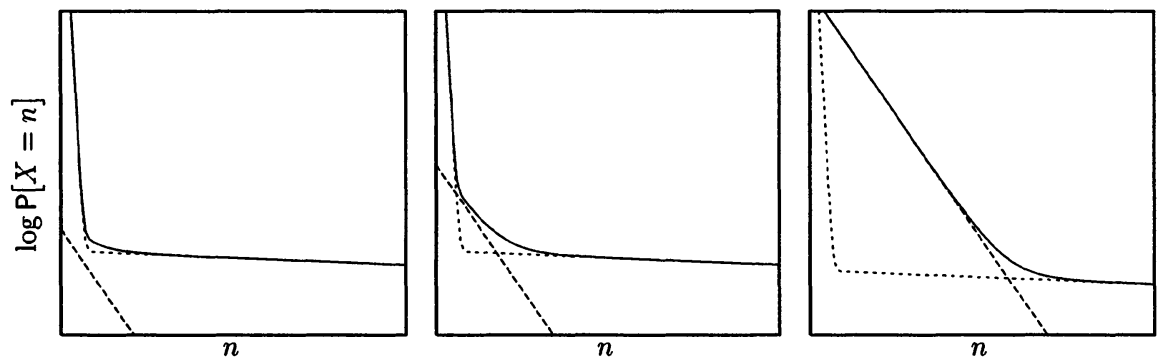

Figure 3 effect of adding a third phase to a 2-phase distribution $\mathrm{P}[X=n]=\alpha\left(1-x_{3}\right) x_{3}{ }^{n}+(1-\alpha)\left(A\left(1-x_{1}\right) x_{1}{ }^{n}+(1-A)\left(1-x_{2}\right) x_{2}{ }^{n}\right)$ with $A=0.998, x_{1}=0.01, x_{2}=0.99, x_{3}=0.7$ and $\alpha=10^{-4}, 10^{-2}, 0.5$

composed of the weighted sum of a number of geometric phases, just two phases often are dominant. In this illustration the distribution has three geometric phases of various geometric rates $x_{1}, x_{2}, x 3$. The three graphs show, from left to right, the effect of progressively increasing the contribution $\alpha$ of the intermediate rate $\left(x_{3}\right)$ phase while maintaining constant the ratio $1-A: A$ between the weights of the high rate $\left(x_{2}\right)$ phase and the low rate $\left(x_{1}\right)$ phase. In each graph, the contribution of the high and low rate phases is shown by a feint dotted line, the contribution of the intermediate rate phase alone is shown by the line of dashes and the overall distribution is shown by the continuous solid line. When the weight of the intermediate rate phase is small (left hand graph) it has negligible effect whereas when its weight is large (right hand graph) it effectively nullifies the effect of the low rate phase. With moderate weighting (centre graph) the effect of the intermediate rate phase is merely to round the "knee" of the graph. Thus, in each of the cases, just two phases have significant contribution to the overall distribution.

Furthermore, the form of the MMBBP correlation functions (equations (6) and (7)) indicate that only the first two moments of the batch size distribution 
are of great significance. So a 2-phase count distribution should be sufficient to reveal the dominant effects. For given first two moments of the 2-phase distribution the most extreme behaviour is given when the first segment of the graph (figure 3) is vertical. The distribution then becomes the generalised geometric (GGeo) distribution (see equation (11) below) which is completely defined by the first two moments and is, indeed, the least biased distribution given the first two moments.

\section{Modulating Phases}

By argument essentially similar to that for the batch size distributions, it is clear that the modulating process (i.e. the underlying Markov chain) should have just two phases. A 2-phase MMBBP is called a switched batch Bernoulli process (SBBP). A comprehensive analysis of the $\mathrm{SBBP} / \mathrm{G} / 1$ queue is given by Hashida, Takahashi and Shimogawa (1991).

\section{Intensity}

It appears generally true that high traffic intensity leads to extreme behaviour in the queue. So the experimental model should provide for high intensity and, in particular, for one phase having intensity greater than one.

The form of the MMBBP correlation functions indicate that the difference in mean intensity between phases is significant. Thus the experimental model should provide for large difference in intensity between phases.

\subsection{Typical Results}

Table 1 shows typical sets of parameters selected in accordance with the foregoing design scheme.

For each phase $i$ the GGeo count distribution is given by the probability $\lambda_{i}(n)$ of $n$ cells being generated at an epoch

$$
\begin{aligned}
\lambda_{i}(n) & =\frac{1-\eta_{i}-\nu_{i}}{1-\nu_{i}} \delta_{n 0}+\frac{\eta_{i}}{1-\nu_{i}} \nu_{i}\left(1-\nu_{i}\right)^{n} \\
& = \begin{cases}1-\eta_{i} & n=0 \\
\eta_{i} \nu_{i}\left(1-\nu_{i}\right)^{n-1} & n=1,2, \ldots\end{cases}
\end{aligned}
$$

where $\delta$ is the Kronecker delta.

The probabilities $\phi_{i j}$ of transition from phase $i$ to phase $j$ at any epoch are designated conventionally

$$
\phi_{12}=p, \quad \phi_{21}=q, \quad \phi_{11}=1-p, \quad \phi_{22}=1-q .
$$

The factors $\beta_{b i}=1-\eta_{i}-\nu_{i}$ and $\beta=1-p-q$ are useful indicators of correlation. When zero they indicate independence. When $\beta$ be close to 1 the sojourn of the SBBP is long in each phase. Count correlation increases with $\beta$ for any given stationary phase distribution. When $\beta_{b i}$ be close to 1 the 


\begin{tabular}{|c|c|c|c|c|c|c|c|c|}
\hline \multirow[b]{2}{*}{ graph } & \multicolumn{3}{|c|}{ phase 1 , intensity 0.8} & \multicolumn{3}{|c|}{ phase 2 , intensity 1.1} & & \\
\hline & $\eta_{1}$ & $\nu_{1}$ & $\beta_{b 1}$ & $\eta_{2}$ & $\nu_{2}$ & $\beta_{b 2}$ & & \\
\hline$(01)$ & 0.02 & 0.025 & 0.955 & & & & \multirow{4}{*}{$p=0.001$} & \\
\hline$(02)$ & 0.2 & 0.25 & 0.55 & 0.022 & 0.02 & 0.958 & & \\
\hline (03) & 0.4 & 0.5 & 0.1 & & & & & \\
\hline$(04)$ & 0.02 & 0.025 & 0.955 & & & & & \\
\hline$(05)$ & 0.2 & 0.25 & 0.55 & 0.22 & 0.2 & 0.58 & \multirow{5}{*}{$\begin{array}{l}q=0.002 \\
\beta=0.997\end{array}$} & $*$ \\
\hline$(06)$ & 0.4 & 0.5 & 0.1 & & & & & $*$ \\
\hline$(07)$ & 0.02 & 0.025 & 0.955 & & & & & \\
\hline$(08)$ & 0.2 & 0.25 & 0.55 & 0.44 & 0.4 & 0.16 & & $*$ \\
\hline (09) & 0.4 & 0.5 & 0.1 & & & & & $*$ \\
\hline (10) & 0.02 & 0.025 & 0.955 & & & & \multirow{4}{*}{$p=0.03$} & \\
\hline (11) & 0.2 & 0.25 & 0.55 & 0.022 & 0.02 & 0.958 & & \\
\hline (12) & 0.4 & 0.5 & 0.1 & & & & & \\
\hline (13) & 0.02 & 0.025 & 0.955 & & & & & \\
\hline (14) & 0.2 & 0.25 & 0.55 & 0.22 & 0.2 & 0.58 & \multirow[t]{2}{*}{$q=0.06$} & \\
\hline (15) & 0.4 & 0.5 & 0.1 & & & & & \\
\hline (16) & 0.02 & 0.025 & 0.955 & & & & \multirow[t]{3}{*}{$\beta=0.91$} & \\
\hline (17) & 0.2 & 0.25 & 0.55 & 0.44 & 0.4 & 0.16 & & \\
\hline (18) & 0.4 & 0.5 & 0.1 & & & & & \\
\hline (19) & 0.02 & 0.025 & 0.955 & & & & \multirow{4}{*}{$p=0.333$} & \\
\hline (20) & 0.2 & 0.25 & 0.55 & 0.022 & 0.02 & 0.958 & & \\
\hline (21) & 0.4 & 0.5 & 0.1 & & & & & \\
\hline$(22)$ & 0.02 & 0.025 & 0.955 & & & & & \\
\hline (23) & 0.2 & 0.25 & 0.55 & 0.22 & 0.2 & 0.58 & \multirow[t]{2}{*}{$q=0.666$} & \\
\hline (24) & 0.4 & 0.5 & 0.1 & & & & & \\
\hline$(25)$ & 0.02 & 0.025 & 0.955 & & & & \multirow[t]{3}{*}{$\beta=0.001$} & \\
\hline (26) & 0.2 & 0.25 & 0.55 & 0.44 & 0.4 & 0.16 & & \\
\hline (27) & 0.4 & 0.5 & 0.1 & & & & & \\
\hline
\end{tabular}

For the sets of parameters which are marked * there is no corresponding batch renewal process which is proper.

Table 1 Parameters of the SBBP for graphs 01-27 - mean intensity 0.9

variability of counts is high during phase $i$. Interval correlation increases with $\beta_{b i}$.

Table 1 shows $3 \times 3 \times 3$ parameter sets, arranged in decreasing magnitude of $\beta, \beta_{b 2}, \beta_{b 1}$, and the corresponding graphs of queue length distribution (q.l.d.) are shown in figure 4 . The experimental results are typical of those obtained from sets of parameters selected in accordance with the design scheme described above. 

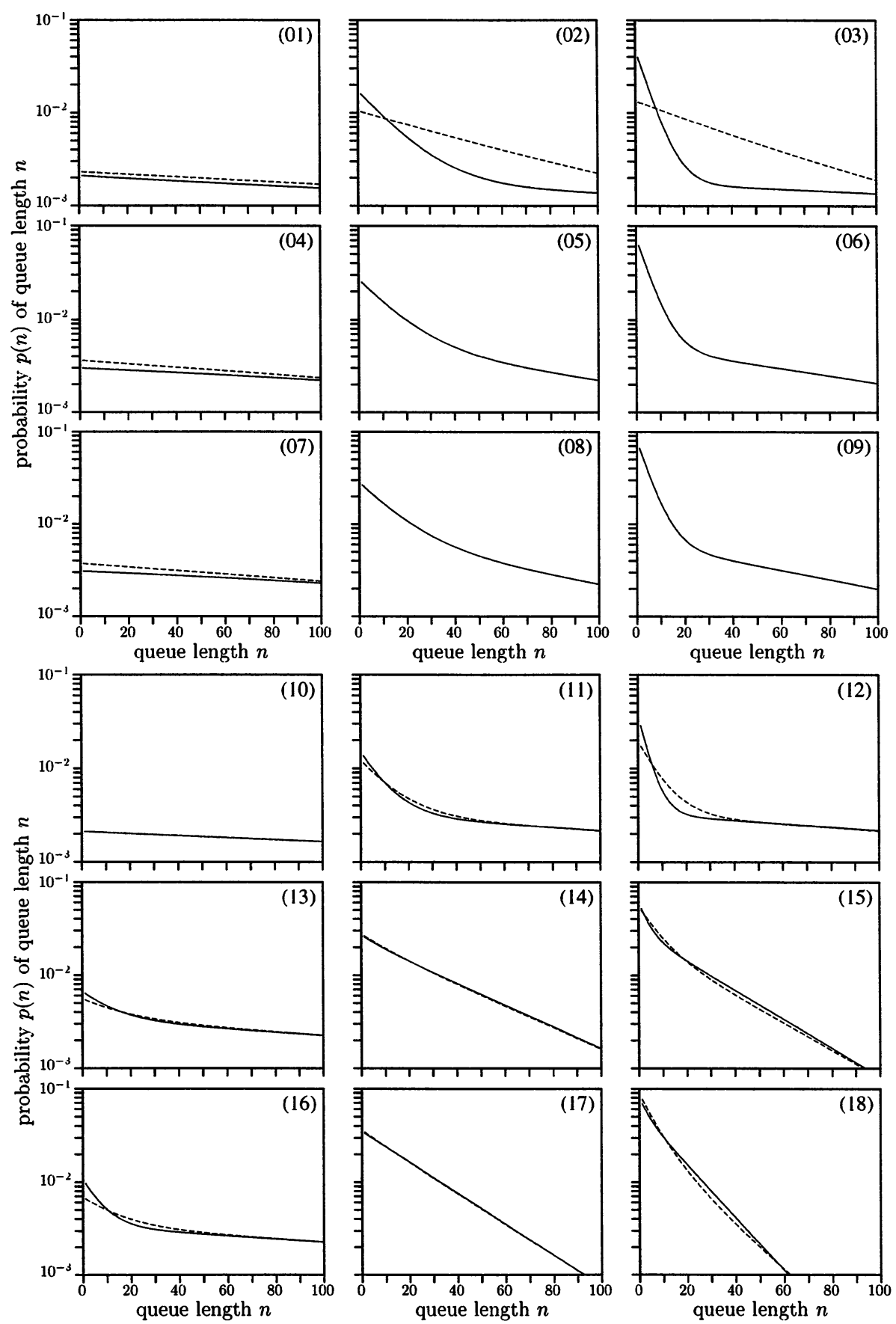

Figure 4 Queue length distributions for SBBP and for the autocorrelation of the SBBP (part 1 - graphs 01-18) 

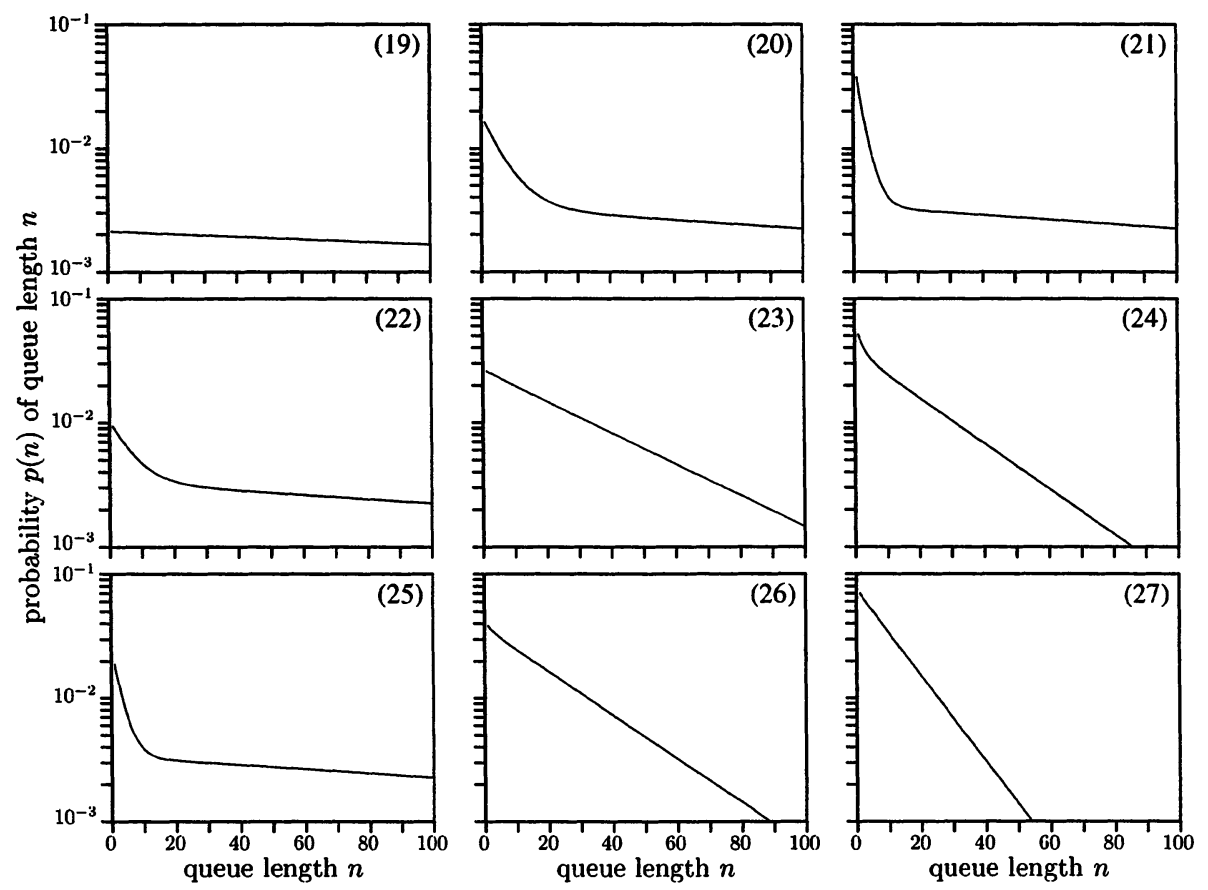

Figure 4 Queue length distributions for SBBP and for the autocorrelation of the SBBP (part 2 - graphs 19-27)

The form of each queue length distribution is

$$
p(n)= \begin{cases}1-\lambda & n=0 \\ \lambda A\left(1-x_{1}\right) x_{1}{ }^{n}+\lambda(1-A)\left(1-x_{2}\right) x_{2}{ }^{n} & n=1,2 \ldots\end{cases}
$$

(i.e. a modified weighted sum of two geometrics with rates $x_{1}, x_{2}$ ) both for the SBBP/D/1 queue and for the GIG $/ D / 1$ queue fed by the corresponding batch renewal process with the same correlation as the SBBP i.e. the q.l.d. attributable to the correlation alone of the SBBP.

In each graph of figure 4, the solid line shows the SBBP/D/1 q.l.d. and the dashed line shows the $\mathrm{GI}^{\mathrm{G}} / \mathrm{D} / 1$ q.l.d. No dashed line is shown on graphs 05 , 06, 08 and 09 because the corresponding batch renewal process is not proper and the validity of improper batch renewal processes is not known.

Most of the graphs have the characteristic "knee" form (c.f. graph 12) with a steep first segment (corresponding to the smaller geometric rate $x_{1}$ ) followed by a flatter second segment (corresponding to the larger geometric rate $x_{2}$ ). In some graphs (viz. 14, 17, 18) the rates $x_{1}, x_{2}$ are of similar magnitude and the "knee" is not very apparent. In some other graphs (viz. 01, 04, 07, 10, 19) the first segment does not appear because the corresponding weight $A$ is small. In the last group of graphs (19-27) the two q.l.d.'s are so close in magnitude 
(agreement to several significant digits) that the difference between solid line and the dashed line cannot be discriminated. However, in every case, the smaller geometric rate $x_{1}$ of the $\mathrm{SBBP} / \mathrm{D} / 1$ q.l.d. is smaller than that in the $\mathrm{GI}^{\mathrm{G}} / \mathrm{D} / 1$ q.l.d.

In the typical result (exemplified by graph 12), the smaller rate (steeper first segment) in the SBBP/D/1 q.l.d. clearly implies lower waiting time, less jitter and, extrapolating to the finite buffer case, lower cell loss rate as compared with the distribution attributable to the correlation alone. In other words, the SBBP yields optimistic results. On the other hand, the cases (c.f. graphs 01, 04, 07, 10, 19) in which the SBBP/D/1 q.l.d. be pessimistic do not correspond to acceptable operating conditions: jitter would be high and, extrapolating to the finite buffer case, cell loss rate would be high.

\section{ANALYSIS}

It is inherently difficult to argue a contention such as Markov modulated process models are likely to yield results which are more optimistic than justified by the traffic correlation alone.

The term "Markov modulated process" is not well defined. Other processes, such as DBMAP's and discrete time Markov renewal processes, can also be described as Markov modulated processes (see section 2). Even the standard for comparison (the batch renewal process) can be described as Markov modulated processes: how could the standard be "more optimistic" than itself?

Even were it possible to find a generally acceptable restrictive definition of "Markov modulated process" and to identify neccessary and sufficient conditions under which the (restricted) Markov modulated process yielded optimistic results, there remains the more problematic issue of assessing how likely those conditions are to occur in practice, i.e. positing the type of Markov modulated process and parameter values which might be employed by a performance analyst.

It is supposed that 1) a Markov modulated process would be used when the traffic exhibited positive correlation of counts and of intervals and 2) the model would have only the minimum features consistent with the traffic being modelled. This second supposition is taken to imply in particular that (c.f. Figure 3) the lowest rate in the q.l.d. have sufficient weight to feature in the q.l.d. (i.e. not as in the third graph of Figure 3 and graphs 01, 04, 07, 10, 19 of Figure 4). Under these suppositions, the contention is upheld if the following proposition be true in most cases of practical interest.

Proposition The largest pole of the MMBBP/D/1 q.I.d. generating function is larger than that of the corresponding $\mathrm{GI}^{\mathrm{G}} / \mathrm{D} / 1$ queue i.e. that attributable to the MMBBP traffic correlation alone. 
(Observe that the geometric rates of the q.l.d. are the reciprocals of the poles of the q.l.d. generating function.)

The first part of this section is an argument that the proposition be plausible for a sterotype process. The second subsection presents statistics from a Monte Carlo experiment which was designed both to address potential weaknesses in the first experiment (section 3) and also to provide some estimate for the probability that the proposition be true.

\subsection{Qualitative Analysis}

This section presents an analysis of the relationships which might be expected to obtain between the spectra of the generating functions for the queue length distributions of the queue fed by a MMBBP and the $\mathrm{GI}^{\mathrm{G}} / \mathrm{D} / 1$ queue fed by the batch renewal process which has the same measures of traffic correlation.

The stereotype generating function (say) $f(z)$ is assumed to be that for a weighted sum of geometrics and, therefore, to have the general form indicated by Figure 5 for real $z$ and real $f(z)$. In this form, $f(z)$ is non-decreasing

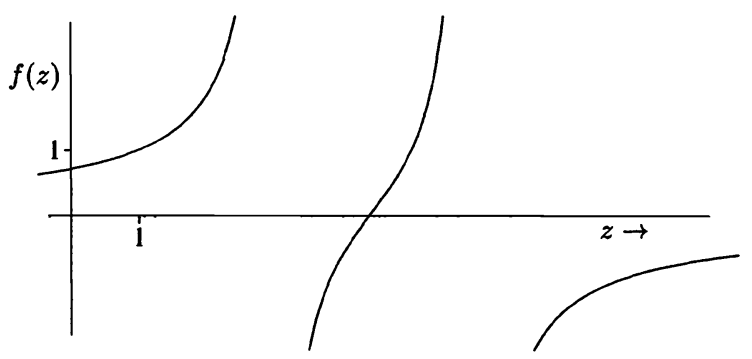

Figure 5 graph of stereotype of generating function

everywhere except at poles. The poles of $f(z)$ are real and, in the region of a pole $z_{0}, f(z)$ has the expansion

$$
f(z)=\frac{-r}{z-z_{0}}-g(z)
$$

where the residue $r>0$ at the pole $z_{0}$ and the remainder $g(z) \geq 0$ in the region of the pole.

Define $D(\theta, z)=\operatorname{det}(\theta \mathbf{I}-\boldsymbol{\Lambda}(z) \boldsymbol{\Phi})$. Let $z_{0}$ be the largest pole of $\boldsymbol{\Lambda}(z)$, i.e. the largest amongst the poles of $\Lambda_{1}(z), \ldots, \Lambda_{J}(z)$, and assume that $z_{0}$ be a pole of $\Lambda_{1}(z)$ only. Let $z_{1}$ be the largest pole of $P(z)$, i.e. the largest zero of $D(z, z)$, let $z_{2}$ be the largest pole of $L(z)$, i.e. the largest zero of $D(1, z)$, let $z_{3}$ be the largest zero of $C(z)=1-A(1 / z) B(z)$ and $z_{4}$ be the largest pole of $B(z)$.

It is to be expected that the relationships $z_{2}<z_{1}<z_{0}$ and $z_{2}<z_{3}<z_{4}$ would apply and then the point of interest is the conditions for which $z_{4}<z_{1}$. 
Consider $D(\theta, z)=\operatorname{det}(\theta \mathbf{I}-\mathbf{\Lambda}(z) \mathbf{\Phi})$. When $D(\theta, z)=0$, at (say) $z=z_{\theta}$, then

$$
\begin{aligned}
& \Lambda_{1}\left(z_{\theta}\right)=\frac{\theta}{\phi_{11}} \frac{\theta^{J-1}+c_{1}\left(z_{\theta}\right) \theta^{J-2}+\cdots+c_{J-1}\left(z_{\theta}\right)}{\theta^{J-1}+d_{1}\left(z_{\theta}\right) \theta^{J-2}+\cdots+d_{J-1}\left(z_{\theta}\right)} \\
& \text { where } c_{1}(z)=-\phi_{22} \Lambda_{2}(z)-\cdots-\phi_{J J} \Lambda_{J}(z) \text {, } \\
& c_{2}(z)=\left|\begin{array}{cc}
\phi_{22} & \phi_{23} \\
\phi_{32} & \phi_{33}
\end{array}\right| \Lambda_{2}(z) \Lambda_{3}(z)+\cdots \\
& \cdots+\left|\begin{array}{cc}
\phi_{J-1, J-1} & \phi_{J-1, J} \\
\phi_{J, J-1} & \phi_{J J}
\end{array}\right| \Lambda_{J-1}(z) \Lambda_{J}(z), \\
& c_{J-1}(z)=(-1)^{J-1}\left|\begin{array}{llr}
\phi_{22} & \ldots & \phi_{2 J} \\
\ldots \ldots & \ldots & \ldots \\
\phi_{J 2} & \ldots & \phi_{J J}
\end{array}\right| \Lambda_{2}(z) \ldots \Lambda_{J}(z) \\
& \text { and } \quad d_{1}(z)=-\frac{1}{\phi_{11}}\left|\begin{array}{cc}
\phi_{11} & \phi_{12} \\
\phi_{21} & \phi_{22}
\end{array}\right| \Lambda_{2}(z)-\cdots-\frac{1}{\phi_{11}}\left|\begin{array}{cc}
\phi_{11} & \phi_{1 J} \\
\phi_{J 1} & \phi_{J J}
\end{array}\right| \Lambda_{J}(z) \text {, } \\
& d_{J-1}(z)=\frac{(-1)^{J-1}}{\phi_{11}} \operatorname{det} \Phi
\end{aligned}
$$

The largest zero $z_{\theta}$ of $D(\theta, z)$ may be assumed to lie near $z_{0}$, the largest pole of $\Lambda(z)$. Observe firstly that $\Lambda_{2}\left(z_{\theta}\right), \ldots, \Lambda_{J}\left(z_{\theta}\right)$ are all negative because $z_{\theta}$ is larger than the largest pole of any of the functions $\Lambda_{2}(z), \ldots, \Lambda_{J}(z)$ and all those functions are assumed to conform to the stereotype. Secondly, $\boldsymbol{\Phi}$ is assumed to be diagonally dominant. Under these conditions, all the coefficients $c_{1}\left(z_{\theta}\right), \ldots, c_{J-1}\left(z_{\theta}\right)$ and $d_{1}\left(z_{\theta}\right), \ldots, d_{J-1}\left(z_{\theta}\right)$ are positive and also $d_{i}\left(z_{\theta}\right) \approx$ $c_{i}\left(z_{\theta}\right)$ Therefore, the location of the largest zero of $D(\theta, z)$ may estimated by the (apparently crude) approximation that, at that point, $\Lambda_{1}(z) \approx \theta / \phi_{11}$. Then, for $\Lambda_{1}(z)$ of the form of expression (13), the largest poles $z_{1}$ and $z_{2}$ of $P(z)$ and $L(z)$ may be estimated by

$$
z_{1} \approx z_{0}-\frac{r}{g\left(z_{0}\right)+z_{0} / \phi_{11}} \quad \text { and } \quad z_{2} \approx z_{0}-\frac{r}{g\left(z_{0}\right)+1 / \phi_{11}} .
$$

Thus $z_{2}<z_{1}<z_{0}$.

To judge the accuracy of those estimates, consider a SBBP, for which $D\left(\theta, z_{\theta}\right)=0$ yields

$$
\Lambda_{1}\left(z_{\theta}\right)=\frac{\theta^{2}-\phi_{22} \Lambda_{2}\left(z_{\theta}\right) \theta}{\phi_{11} \theta-\left(\phi_{11} \phi_{22}-\phi_{12} \phi_{21}\right) \Lambda_{2}\left(z_{\theta}\right)}
$$

and thence

$$
z_{1} \approx z_{0}-\frac{r}{g\left(z_{0}\right)+z_{0} \frac{z_{0}-\phi_{22} \Lambda_{2}\left(z_{0}\right)}{\phi_{11} z_{0}-\beta \Lambda_{2}\left(z_{0}\right)}}
$$


which is close to the approximation given by (15) when $\phi_{11} \phi_{22}$ is close to $\beta$. But $\phi_{11} \phi_{22}=\beta+(1-\beta)^{2} \phi_{1} \phi_{2}$ and clearly $\phi_{1} \phi_{2} \leq 1 / 4$. If, for example, $\beta=1 / 2$ then $\phi_{11} \phi_{22}$ exceeds $\beta$ by $6.25 \%$ at most. If, more realistically, $1-\beta<10^{-3}$ the relative difference is negligible.

For the queue fed by the corresponding batch renewal process, the largest pole of the q.l.d. generating function is seen (Kouvatsos and Fretwell 1994) to be the largest zero of $1-A(1 / z) B(z)$. If $A(\omega)$ is proper it has no singularities inside the unit disk $|\omega| \leq 1$ and so $0 \leq A(1 / z) \leq 1$ for $z>1$. Then, assuming $B(z)$ conforms to the stereotype generating function, the largest zero $z_{3}$ of $1-A(1 / z) B(z)$ must be near to but less than the largest pole $z_{4}$ of $B(z)$.

Equating (6) with (9) and (7) with (10) leads (see Kouvatsos and Fretwell 1996) to explicit expressions for $A(\omega)$ and $B(z)$. In particular

$$
\begin{aligned}
& B(z)=\frac{\lambda \phi(\mathbf{I}-\boldsymbol{\Lambda}(z) \Phi)^{-1} \boldsymbol{\Lambda}(z) \mathbf{1}-\lambda \phi(\mathbf{I}-\boldsymbol{\Lambda}(0) \Phi)^{-1} \boldsymbol{\Lambda}(0) \mathbf{1}}{\lambda \phi(\mathbf{I}-\boldsymbol{\Lambda}(z) \Phi)^{-1} \boldsymbol{\Lambda}(z) \mathbf{1}-M} \\
& \text { where } \quad M=\lim _{z \rightarrow 1}\left(\lambda \phi(\mathbf{I}-\Lambda(z) \Phi)^{-1} \boldsymbol{\Lambda}(z) \mathbf{1}-\frac{1}{1-z}\right)-\frac{1}{\lambda} \phi \Lambda^{2} \mathbf{1} \\
& =\lim _{\omega \rightarrow 1}\left(\frac{1}{\lambda} \phi \mathbf{\Lambda}(\mathbf{I}-\omega \mathbf{\Phi})^{-1} \Lambda \mathbf{1}-\lambda \frac{1}{1-\omega}\right)-\frac{1}{2 \lambda} \phi \Lambda^{\prime \prime}(1) \mathbf{1}
\end{aligned}
$$

Now $z_{2}$ is the largest pole of $\lambda \boldsymbol{\phi}(\mathbf{I}-\boldsymbol{\Lambda}(z) \Phi)^{-1} \Lambda(z) \mathbf{1}$ which (c.f. expression (13)), in the neighbourhood of $z=z_{2}$, takes the form

$$
\lambda \phi(\mathbf{I}-\boldsymbol{\Lambda}(z) \boldsymbol{\Phi})^{-1} \boldsymbol{\Lambda}(z) \mathbf{1}=-\frac{r_{2}}{z-z_{2}}-g_{2}(z)
$$

and

$$
B(z) \approx \frac{r_{2}+\left(z-z_{2}\right)\left(g_{2}\left(z_{2}\right)+\lambda \phi(\mathbf{I}-\Lambda(0) \Phi)^{-1} \Lambda(0) \mathbf{1}\right)}{r_{2}+\left(z-z_{2}\right)\left(g_{2}\left(z_{2}\right)+M\right)} .
$$

So the largest pole $z_{4}$ of $B(z)$ is near $z_{2}$ at

$$
z_{4} \approx z_{2}-\frac{r_{2}}{g_{2}\left(z_{2}\right)+M}
$$

Whenever $M$ be non-negative, $z_{4}<z_{2}$ and so $z_{4}<z_{1}$, in support of the proposition. However, the proposition may still be true even if $M$ be negative. Using the second form in the defining equation (17) shows that for $\Phi$ semisimple*

$$
M=\frac{1}{\lambda} \sum_{r=2}^{J} \frac{1}{1-\mu_{r}} \phi \Lambda X_{r} Y_{r} \Lambda 1-\frac{1}{2 \lambda} \phi \Lambda^{\prime \prime}(1) 1
$$

where the $\mu_{r}, \boldsymbol{X}_{r}, \boldsymbol{Y}_{r}$ are respectively the sub-dominant eigenvalues and corresponding right and left eigenvectors of $\Phi$. Clearly, for any particular

\footnotetext{
* It may be argued that a randomly selected matrix is almost surely semi-simple.
} 
stationary phase distribution $\phi$ and distribution of counts in the phases, $M$ increases with increase in the largest sub-dominant eigenvalue $\beta=\mu_{2}$. Loosely, the more strongly diagonally dominant be the phase transition matrix $\Phi$ the more likely is $M$ to be large enough for $z_{4}<z_{1}$ to be true.

\subsection{Monte Carlo Experiment}

This subsection describes a Monte Carlo experiment to assess the likelihood that the proposition be true.

A second purpose for this experiment was to address possible weaknesses in the design of the first experiment. It is conceivable that the results of the first experiment could be dominanted by the extreme forms chosen (2-phase MMBBP with GGeo counts and high mean intensity) or by unwitting bias in selection of parameters. For the distribution of counts in each phase, instead of using the extremal GGeo form the distribution could be a sum of (simple) geometrics. But then, because each sum of geometrics may be decomposed into its constituent phases, it becomes sufficient to consider (simple) geometric distributions only.

For each of 2-, 3- and 6-phase models, 4000 MMBBP's were generated randomly: for each MMBBP the phase transition matrix entries were taken randomly from a uniform distribution and then each row of the matrix was normalised; for each MMBBP the mean intensity for each phase was taken randomly from a uniform distribution and scaled (5 times) to give over all mean rates (i.e. with respect to the stationary phase distribution for the particular MMBBP) of $0.1,0.3,0.5,0.7$ and 0.9. For each MMBBP with each value of mean rate, the largest poles of the $\mathrm{MMBBP} / \mathrm{D} / 1$ and corresponding batch renewal process q.l.d. generating functions were computed and compared. Then all the (60000) computations and comparisons were repeated after biasing each transition matrix to ensure diagonal dominance: the bias was applied to each row by halving each off-diagonal entry and increasing the diagonal entry in compensation. The effect of selecting stochastic matrices randomly and then applying this bias is precisely the same as selectly randomly from the set of diagonally dominant matrices.

Table 2 shows the proportion of cases in which the proposition was true. Observe that, for randomly selected matrices, the proportion which are diagonally dominant is 1 in $J^{J}$, where $J$ is the degree of the matrices. In Table 2 the "unbiased" column shows that the proposition is true for a much larger proportion of MMBBP's than the proportion of phase transition matrices which are diagonally dominant. Observe also that, from the "biased" column, diagonal dominance is not sufficient to guarantee the truth of the proposition.

The proposition is likely to be true for MMBBP's with a small number of modulating phases and for MMBBP's with diagonally dominant phase transi- 


\begin{tabular}{ccrr} 
degree & $\lambda$ & unbiased & biased \\
\hline 2 & 0.1 & $81 \%$ & $>99 \%$ \\
& 0.3 & $81 \%$ & $>99 \%$ \\
& 0.5 & $81 \%$ & $>99 \%$ \\
& 0.7 & $81 \%$ & $>99 \%$ \\
& 0.9 & $81 \%$ & $>99 \%$ \\
3 & 0.1 & $80 \%$ & $>99 \%$ \\
& 0.3 & $80 \%$ & $>99 \%$ \\
& 0.5 & $80 \%$ & $>99 \%$ \\
& 0.7 & $80 \%$ & $>99 \%$ \\
& 0.9 & $80 \%$ & $>99 \%$ \\
6 & 0.1 & $46 \%$ & $88 \%$ \\
& 0.3 & $46 \%$ & $88 \%$ \\
& 0.5 & $46 \%$ & $89 \%$ \\
& 0.7 & $46 \%$ & $93 \%$ \\
& 0.9 & $45 \%$ & $94 \%$
\end{tabular}

Table 2 Proportion of cases supporting the proposition

tion matrices. Mean intensity has little effect on the truth of the proposition, especially for smaller number of modulating phases.

The results strangly suggest that the MMBBP is likely to yield optimistic results, under the supposed conditions of positive traffic correlation of counts and intervals and the minimum model features consistent with the modelled traffic, i.e. the "biased" column of Table 2.

\section{CONCLUSIONS AND OTHER REMARKS}

It is empirically evident that Markov modulated processes are likely to give optimistic values for cell loss rate, delay, etc. for given measures of count correlation and intervals correlation. The conclusion to be drawn is that Markov modulated processes should not be used to model traffic - unless, of course, the cross-correlation between counts and intervals is known to correspond to that of the model Markov modulated process.

The experiment and analysis which is reported in this paper was designed to yield results of general applicability and, therefore, the authors contend that the results imply that the statement above be broadly true.

There are some regions of MMBBP parameterisation for which a proper batch renewal process does not exist. Formal analysis is impossible without means to educe the queue length distribution attributable to the traffic correlation alone. One approach which is being considered is to seek sums and differences of processes for which those sums or differences can be shown to be minimally biased given the traffic correlation alone. 


\section{Aknowlegement}

The authors wish to thank three referees for their constructive comments and suggestions on an earlier version of this paper.

\section{REFERENCES}

Sriram, K. and Whitt W. (1986) Characterizing Superposition Arrival Processes in Packet Multiplexers for Voice and Data, IEEE JSAC 4(6), 833-846

Gusella, R. (1991) Characterizing the Variability of Arrival Processes with Indexes of Dispersion, IEEE JSAC 9(2), 203-211

Heffes, H. and Lucantoni, D.M. (1986) A Markov Modulated Characterization of Packetized Voice and Data Traffic and Related Statistical Multiplexer Performance, IEEE JSAC 4(6), 856-868

Kouvatsos, D.D.and Fretwell, R.J. (1994) Discrete Time Batch Renewal Processes with Application to ATM Switch Performance', Proc. 10th. UK Computer and Telecomms. Performance Eng. Workshop (ed. Jane Hillston et al.), Edinburgh University Press, 187-192

Kouvatsos, D.D.and Fretwell, R.J. (1995) Closed Form Performance Distributions of a Discrete Time GI ${ }^{G} / D / 1 / N$ Queue with Correlated Traffic, Enabling High Speed Networks (ed. S. Fdida and R.O. Onvural), IFIP Publication, Chapman and Hall, ISBN 041273250 5, 141-163

Kouvatsos, D.D.and Fretwell, R.J. (1996) Batch Renewal Process: Exact Model of Traffic Correlation, High-Speed Networking for Multimedia Applications (ed. W. Effelsberg), Kluwer Academic Publishers, ISBN 079239681 2, 285-304

Hashida, O. Takahashi, Y. and Shimogawa, S. (1991) Switched Batch Bernoulli Process (SBBP) and the Discrete-Time SBBP/G/1 Queue with Application to Statistical Multiplexer Performance, IEEE JSAC 9(3), 394-401 


\section{BIOGRAPHY}

Rod Fretwell received the BSc in Mathematics from the University of Sheffield in 1966 and the MSc degree in Computer Science from Bradford University in 1993. $\mathrm{He}$ is currently a $\mathrm{PhD}$ research student and a research assistant in Department of Computing, University of Bradford. His research interests include queueing theory and ATM traffic Modelling and Characterisation.

Demetres Kouvatsos received the BSc degree in Mathematics from Athens National University in 1970, the MSc degree in Statistics from Victoria University of Manchester in 1971 and the $\mathrm{PhD}$ degree in Computation from UMIST, Institute of Science and Technology, University of Manchester in 1974. He is currently a Professor in Computer Systems Modelling, Department of Computing, University of Bradford. Since early 80's, he pioneered new and cost-effective methodologies for the approximate analysis of arbitrary queueing network models (QNMs) of computer and communication systems. The methodologies are based on the information theoretic principles of maximum entropy and minimum relative entropy, asymptomatic approximation techniques, queueing theoretic concepts and batch renewal processes. He has directed several EPSRC, UK and industrial research projects and is the author or co-author of many papers in the performance modelling and analysis field. He acted as the Chairman of the first four IFIP Workshops on Performance Modelling and Evaluation of ATM Networks (199396) and served as a Co-Chairman of the 3rd International Workshop in"Queueing Networks with Finite Capacity" (1995). His latest technical interests include ATM traffic characterisation, analytic methodologies for discrete-time QNMs and performance evaluation of ATM switch architectures. His professional associations include IFIP Working Group WG 6.3 on the Performance of Computer Networks, Performance Engineering Group of BCS and Royal Statistical Society, UK. 